\title{
An Energy Measurement Based Collision Resolution Protocol*
}

\author{
Sanjeev Khanna ${ }^{\mathrm{a}}$, Saswati Sarkar ${ }^{\mathrm{b}}$, and Insik Shin ${ }^{\mathrm{a}}$
}

${ }^{a}$ Dept. of Computer and Information Science, University of Pennsylvania, PA, USA.

${ }^{\mathrm{b}}$ Dept. of Electrical and Systems Engineering, University of Pennsylvania, PA, USA.

A central problem in wireless packet networks is the efficient resolution of collisions among nodes with independent transmission schedules. Natural metrics for measuring the efficiency of any collision resolution scheme include total time taken for collision resolution, average participation time of each transmitter during contention resolution, average number of transmissions made by a transmitter, and average power consumption per a transmitter. While it is straightforward to design a centralized protocol that optimizes along each of these metrics, the challenge is in designing distributed protocols that are efficient along each one of these metrics. No existing approach performs well along all these metrics simultaneously. In this paper, we present EMCRR, a novel randomized distributed collision resolution protocol that is based on power control and energy measurement techniques. We study the performance of EMCRR both analytically and empirically. Our analytical and empirical results closely match each other and show that EMCRR compares very favorably to all existing schemes.

\section{Introduction}

Wireless nodes exchange information through low bandwidth multiple access radio spectrum. Users in transmission range of each other cannot transmit messages simultaneously. At the same time users not in the same neighborhood can use the transmission medium simultaneously. A key challenge in wireless systems is to resolve this space dependent contention efficiently. Space dependent contention can be mitigated if users have locally unique transmission channels. For this purpose, distinct frequencies(codes) are assigned to contending users for FDMA(CDMA) based multiple access scheme. However, use of multiple channels cannot eliminate the space dependent contention. This is because wireless nodes have single radios and one radio can receive one message or transmit one message, but cannot perform multiple receptions and transmissions simultaneously. So, if multiple messages reach a node at the same time, all of them are wiped out and also a message is rejected if it reaches the node, while the node is in the transmission mode. Both events are termed "collision." A user often has no knowledge of its neighbors transmission schedules, and decides its own transmission schedule independently. As a result, collisions are inevitable in any packet-radio network with distributed control. All wireless transmission protocols need to recover from such collisions. The objective of this 
paper is to provide a new collision resolution approach using power control and energy measurement techniques.

\subsection{Performance Metrics}

We first identify the desirable properties of a collision resolution protocol in context of the current wireless network requirements. Clearly, for any practical implementation, a collision resolution protocol needs to be inherently distributed, i.e., nodes take contention decisions without any centralized coordination. Considering bandwidth and power are typical limited resources in wireless networks, next, we consider the following performance metrics for a collision resolution approach as follows: (1) expected total time consumed in the collision resolution process, (2) average participation time of a transmitter, (3) average number of transmissions per transmitter, and (4) average power consumption per transmitter. In addition, collision resolution approaches should be simple as wireless nodes typically have limited memory and processor power as well.

\subsection{Existing Collision Resolution Approaches}

We first briefly review the existing collision resolution schemes. Several wireless medium access protocols including IEEE 802.11 use a random backoff based collision resolution[9]. Whenever there is a collision, a node backs off for a random amount of time, and then re-attempts once again, and the backoff interval increases exponentially with each reattempt. Tree based collision resolution approach has been suggested in $[2,3,8]$. Whenever there is a collision, all contending nodes randomly split into two subsets. The different subsets attempt transmission in different slots. Further splitting occurs if there are further collisions. A deterministic tree splitting approach has been proposed in CARMA-MC of [6]. Here, the splitting occurs in accordance with ID announcements from the receiver. In the collision multiplicity based splitting of CRAI in [5], the receiver estimates the number of colliding nodes from the received energy, and broadcasts this to the transmitters. The transmitters decide the splitting probability based on the collision multiplicity. We will show later that our proposed approach is substantially better than all the existing ones with respect to all the four metrics.

\subsection{Our Collision Resolution Approach}

We propose a new collision resolution approach which we call EMCRR (Energy Measurement based Collision Resolution in Rounds). The basic intuition behind our approach can be outlined as follows. Collision resolution takes place in rounds. Whenever there is a collision, a receiver node can estimate the number of colliding nodes from the received power, e.g., using Standard circuits[11]. It broadcasts this estimate back to the colliding nodes. The first collision resolution round length is determined as a function of this estimate. The colliding nodes choose their re-attempt slots randomly in the resolution round. At the end of the first re-attempt round, the receiving node broadcasts the slot numbers which had successful re-attempts and the total number of remaining contenders. A colliding node can find out whether its attempt was successful or not from this information. A node that fails in the current round reattempts in the next round. This repeats till each node succeeds. 


\subsection{Performance Comparison}

EMCRR attains good performance w.r.t. all four metrics. For (1) the average total collision resolution interval, we prove the performance of EMCRR is upper-bounded by $2.78 k$ slots, where $k$ is the total number of contenders. So far, the best performance has been attained by the CRAI protocol of [5] and the number is $3.74 k$ for $k$ nodes. Simulation results indicate that the performances of the other approaches $[2,3,6,8,9]$ exceed that of EMCRR by at least a factor of 1.7 for broad range values of $k$, and the factor increases with increase in $k$. For (2) the maximum participation time of a transmitter, we prove the performance in EMCRR is upper bounded by $4.36 \ln k+.075 k$ with a high probability. Analytical results are not available for the other approaches. Simulation results indicate that there is an improvement by a factor of 1.5 to all the known approaches $[2,3,5,6,8,9]$. For (3) the average number of transmissions, EMCRR has fewer as compared to others for a large number of contenders $(k \geq 64)$. The difference increases with the increase in the number of contenders. We also prove that the performance EMCRR is upper bounded by $e+1$ independent of the number of transmitters, whereas for all other approaches, this number increases with the increase in the number of contenders. For (4) the average power consumption, We observe through simulations that power consumption is at least 2 times more for all the other approaches as long as the number of contenders is greater than $8(k \geq 8)$. Power consumption depends on the average participation times, the average number of transmissions and actual values of power consumed in different activities like transmission, reception, channel monitoring and sleeping. The improvement in power consumption is mainly due to the significant reduction in the average participation time.

The rest of the paper is organized as follows. Section 2 describes our network model, assumptions and the proposed protocol and discusses some salient features of our policy. Section 3 presents our analytical results. Section 4 describes existing collision resolution approaches, and compares our policy with these. We conclude with some directions for future work in Section 5.

\section{The EMCRR Collision Resolution Protocol}

\subsection{Assumptions}

We consider a multichannel symmetric wireless network where the channel is a frequency (code) for a frequency (code) division multiple access. We assume every potential receiver $^{2}$ is assigned a locally unique receiving channel in its two-hop neighborhood. This channel assignment is achievable using a distributed algorithm proposed in [7]. Each node transmits packets to its intended receiver in the receiver' channel. This mechanism ensures only flows sharing the same receiver interfere with each other[6].

The channel access falls into two modes: (a)transmission mode and (b)reception mode. When a node has no packet to transmit, it is in the reception mode. When a node has packets to transmit, it may be in either mode depending on the communication history, the bandwidth allocation policy, the traffic availability, and etc.

We consider a slotted system with synchronized slot boundaries. The receiver sends

\footnotetext{
${ }^{2}$ In general, all nodes may not be potential receivers. Consider a sensor network, where sensors communicate their measurements to some predetermined nodes. In this case the sensors do not serve as receivers, and hence do not need designated channels. However, if all nodes are potential receivers, then all nodes need locally unique channels.
} 
synchronization information initiating a new communication with a control message (i.e., an RTR (Ready-To-Receive) message). The transmitters synchronize their clocks with the receiver on receiving this information. Similar synchronization is performed in many commercial wireless adhoc standards, including bluetooth[1]. We assume the propagation delays are negligible as compared to packet transmission times. This is the case for single hop wireless links, except the satellite communications.

We assume a node can measure the signal strength of a received packet and adjust its transmission power to a certain degree. Signal strength decays as $d^{-r}$, where $d$ is the distance traversed and $r$ is normally close to 4 . We assume that a good estimate of the path loss index $r$ is known at the receiver. When a transmitter transmits a packet to a receiver with signal strength $P_{s}$, the strength of the received signal is $P_{r}$, where

$P_{r}=P_{s} \cdot d^{-r}$. The receiver estimates $d$ from the knowledge of $P_{s}$ and $r$. When a transmitter knows $d$ and wants its receiver to receive a packet with the signal strength $P_{r}$, the transmitter can transmit with the signal strength $P_{s}$, where $P_{s}=P_{r} / d^{-r}$.

When multiple transmitters transmit to the same receiver simultaneously, all the messages collide at the receiver. We assume that the receiver can measure the total received power on the reception of the corrupt messages. If all the contenders transmit their packets with power scaling such that each packet reaches the receiver with the same signal strength $P_{r}$, which is the sum of the powers of all the transmissions. The receiver estimates the number of contenders from the total received power. If $k$ transmitters transmit in all where the received power is $k P_{r}$, the receiver can figure out that $k$ transmitters transmitted.

The distance between a transmitter and the receiver may change during the collision resolution process on account of mobility. But, the transmitters can continuously update this estimate from the strength of the control packets (i.e., feedback packets) at the end of each collision resolution round.

\subsection{Protocol Operation Overview}

We first provide an outline of the protocol operation as illustrated in Figure 1. Similar to IEEE 802.11, the protocol has a 5 phase handshake. The phases are (a) a receiver broadcasts an RTR control message, (b) a transmitter sends back to the receiver an RTS (Ready-To-Send), (c) the receiver responds to the transmitter by sending a CTS (ClearTo-Send) if the packet is received correctly, otherwise collision resolution is used, (d) the transmitter sends a DATA packet after the resolution of collision, (e) and the receiver finishes the communication by sending an ACK. Now, we explain each phase.

\subsection{Receiver-initiated 5 Phase Handshake Protocol}

Our protocol consists of 5 handshakes initiated by a receiver. Among the 5 handshakes, the first three control message exchanges belong to control message exchange phase and the last two belong to data exchange phase. When a node switches to the reception mode, it broadcasts an RTR in its receiving channel starting the control message exchange phase. Since there is only one receiver in the receiving channel and only the receiver sends an RTR, there is no collision for the RTR. This control message is transmitted with a power $P$ which is uniform throughout the system and contains the clock synchronization information. When a transmitter waiting for an RTR in the channel receives the RTR, it synchronizes its clock with the receiver and also measures the signal strength of this RTR message. Then, the transmitter sends to the receiver an RTS control message that 

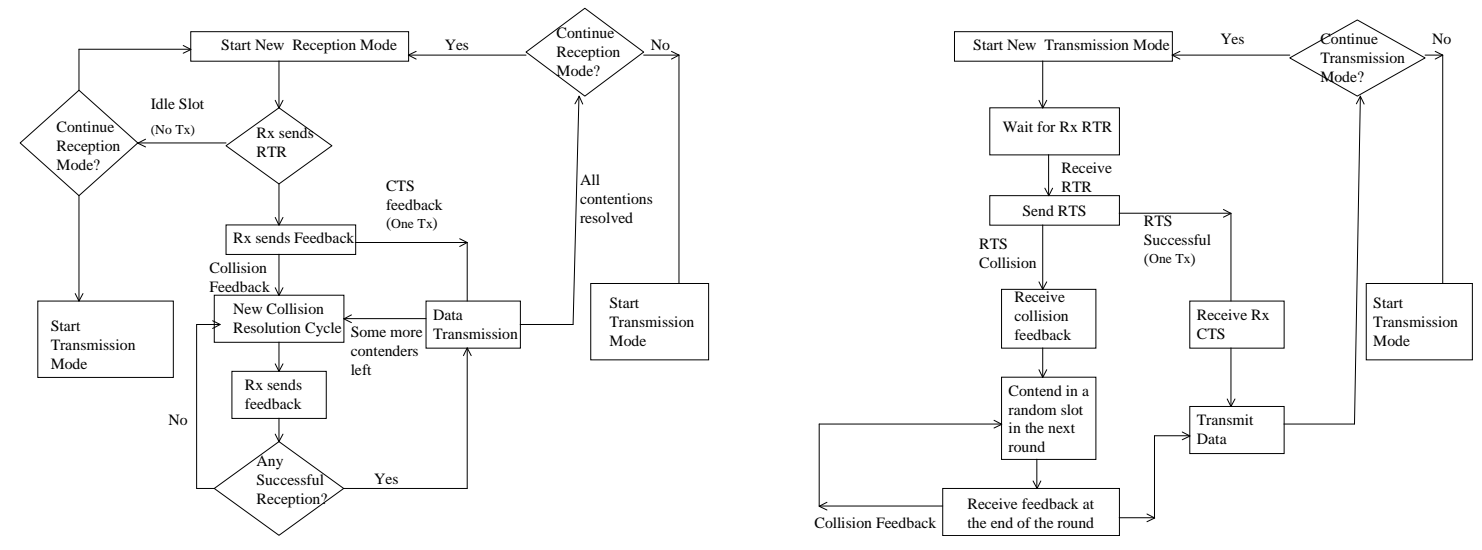

Figure 1. A flowchart for the the Reception (left) and the Transmission (right) Modes. Here, Rx indicates the receiver and Tx indicates the transmitter.

contains the number of packets the transmitter intends to transmit. The transmission power for the RTS message is scaled such that the reception power for this packet after the path loss is $P^{\prime}$, where $P^{\prime}$ is agreed apriori throughout the system.

If there is only one transmitter that transmitted an RTS to the receiver and the RTS is received correctly, then the receiver sends a CTS in the next slot and switches to the data exchange phase. The CTS contains the number of allowed packets. This number is upper bounded by the requested number of packets in the RTS. Like the RTR, there is no collision for the CTS. If a transmitter receives a CTS, it switches to the data exchange phase and starts transmitting data packets.

If multiple transmitters wish to transmit to the receiver (which is normally the case for many applications), all the corresponding RTS collide at the receiver. We discuss the collision resolution now.

\subsection{Collision Resolution Round}

Upon detecting an RTS collision right after an RTR broadcast, the receiver broadcasts a feedback control packet that contains the number of contenders, $k$, participating in the collision. Like an RTR control packet, the feedback packet is transmitted with a power $P$ and contains the clock synchronization. The first collision resolution round starts, and the contenders choose a random integer between 0 and $k-1$ with probability $1 / k$. The choice at any contender is independent of that of the others. If a transmitter chooses $i$. The receiver broadcasts the feedback at the end of the current collision resolution round which is $k$ slot long. The feedback contains the number of contenders participating in the current round. It also contains a list of the slot numbers in which the receiver successfully received RTS and the number of data packets the corresponding transmitters can send. The receiver need not include the identities of the successful transmitters in this packet. A transmitter knows the slot number it attempted, and hence can find out whether or not it succeeded from this feedback. If there are some successful slots, then the receiver and the corresponding transmitters switch to the data exchange phase. We will describe 
the data exchange phase later. A fresh collision resolution round starts after the data exchange phase until there is no collision in the current round.

\subsection{Data Exchange Phase}

The transmitters can compute the data transmission sequences from the information in the feedback packet. For example if a transmitter $N_{j}$ sent RTS successfully in slot $j$ in the current collision resolution round, and there are are $i$ transmitters which succeeded in slots before slot $j$, and $t_{l}$ is the number of packets successful node $N_{l}$ have been permitted to transmit, then transmitter $N_{j}$ transmits data packets in slots $1+\sum_{l=1}^{i} t_{l}$ to $t_{j}+\sum_{l=1}^{i} t_{l}$. It knows the numbers $i, t_{1}, \ldots, t_{i}$ and $t_{j}$ from the feedback packet. The contenders who failed in the previous round can also compute the length of the data transmission phase from the information in the feedback packet. If there were $i$ successes in the previous collision resolution round, then the length of the data transmission phase is $\sum_{l=1}^{i} t_{l}$. Thus the contenders who collided in the previous round, know when a new contention resolution round starts.

\subsection{Discussion}

During a collision resolution round, a contender participates only twice when it transmits an RTS in its randomly chosen slot and when it receives a feedback packet in the last slot of the collision resolution round. It can sleep or participate in other activities in another channel during the other slots. Thus, clearly the participation time of a transmitter is low in this process.

The feedback packets at the end of every round contains information about the number of remaining contenders and the successful slots. Thus collisions do not induce additional feedback. All the tree based approaches $[2,3,5,6,8]$ need feedback after every slot and this increases the total time consumed in the collision resolution.

The protocol is completely distributed. Our scheme is particularly suited to application where there are few receivers.

\section{Analytical Results}

We obtain analytical bounds on the total collision resolution interval, the average participation time of a transmitter and the average number of transmissions in this section. Let there be $k$ initial contenders and let $Z_{i}, 1 \leq i \leq k$, be a random variable that indicates the number of rounds in which the $i$ th transmitter participates before it succeeds. Note that $Z_{i}+1$ is the number of transmissions of transmitter $i$. Let $Z$ denote $\sum_{i=1}^{k} Z_{i}$. Also, let $k_{j}$ denote the number of transmitters that participate in the $j$ th round. Thus the total number of slots that are used in the collision resolution rounds is given by $\sum_{j} k_{j}=\sum_{i=1}^{k} Z_{i}=Z$. This follows because if $m$ transmitters contend in a round then the collision resolution round has $m$ slots. The maximum participation time of any transmitter depends on $Z_{\max }=\max _{i} Z_{i}$. This is because a node transmits and receives once every round it contends. We now derive bounds on the values of $Z$ and $Z_{\max }$.

Let $X_{l}$ be the random variable indicating the number of contenders at the beginning of the $l$ th round. Define $p_{j}(r)$ as the probability that a transmitter succeeds in the $j$ th round given that $X_{j}=r$ and given that it has failed in the previous rounds.

$p_{j}(r)=\left(\begin{array}{l}r \\ 1\end{array}\right)\left(\frac{1}{r}\right)\left(1-\frac{1}{r}\right)^{r-1}$. 
Consider the function $f(q)=(1-1 / q)^{q-1}$. Since $\lim _{q \rightarrow \infty} f(q)=1 / e$ and the function $f(q)$ is monotonically decreasing for all $q \geq 1$, we can conclude that $f(q) \geq 1 / e$, for all $q \geq 1$. It follows that $p_{j}(r) \geq 1 / e$, for all $j, r$. Let $g_{i}(r)$ be the probability that transmitter $i$ collides in round $r$ given that it has collided in all previous rounds.

$\operatorname{Pr}\left(Z_{i} \geq r\right)=\operatorname{Pr}($ Transmitter $i$ collides in the first $r$ rounds $)=\prod_{s=1}^{r} g_{i}(s)$

Denote the event transmitter $i$ colliding in round $r$ as $C_{i, r}$ and the event that transmitter $i$ collides in the first $r-1$ rounds as $C_{i}^{r-1}$.

$$
\begin{aligned}
g_{i}(r) & =\sum_{j=1}^{k} \operatorname{Pr}\left(C_{i r} \mid X_{r}=j, C_{i}^{r-1}\right) \operatorname{Pr}\left(X_{r}=j \mid C_{i}^{r-1}\right) \\
& \leq(1-1 / e) \sum_{j=1}^{k} \operatorname{Pr}\left(X_{r}=j \mid C_{i}^{r-1}\right)\left(\text { since } p_{r}(j) \geq 1 / e\right)=(1-1 / e)
\end{aligned}
$$

From (2) and (3), $\operatorname{Pr}\left(Z_{i} \geq r\right) \leq(1-1 / e)^{r}$

$\mathrm{E} Z_{i}=\sum_{r=0} \operatorname{Pr}\left(Z_{i} \geq r\right) \leq \sum_{r=0}(1-1 / e)^{r}=e$

It follows that $\mathrm{E} Z \leq k e$.

Choosing $r=4.36(\ln k)$, we conclude from (4) that $\operatorname{Pr}\left[Z_{i} \geq 4.36(\ln k)\right] \leq 1 / k^{2}$. Using union bound it follows that $\operatorname{Pr}\left[Z_{\max } \geq 4.36(\ln k)\right] \leq 1 / k$. Thus with probability at least $1-1 / k$, each transmitter is successful within $4.36 \ln k$ rounds.

Theorem 1 The expected number of slots used in the collision resolution rounds is upper bounded by ek and with probability at least $1-1 / k$, all transmitters succeed within $4.36(\ln k)$ rounds.

Note that the feedback at the end of every round consists of successful slot numbers and the number of packets allowed for the corresponding transmitters. We allow 3 bytes for each successful slot, and we assume that every slot can accommodate around 40 bytes (this is the size of the control packets in IEEE 802.11). In all, there are exactly $k$ successful slots. The total number of slots consumed in communicating the feedback is $3 k / 40 .^{3}$ Also, the resolution process needs 3 slots before the first collision resolution round in order to receive the RTR, send the first RTS and receive the collision feedback. Thus from Theorem 1 the expected duration of the collision resolution round is upper bounded by $(e+3 / 40) k+3$ slots, which is approximately $2.78 k$ (the additive constant of 3 can be ignored for reasonably large $k$ ).

Note that a transmitter sends a packet once in each round it contends, and from Theorem 1 it can contend for at most $4.36(\ln k)$ rounds with a high probability (probability greater than $1-1 / k)$. Also, a transmitter needs to receive at most $3 k / 40$ slots of feedback by the previous argument. Again, every transmitter is active for 3 slots before the first

${ }^{3}$ Strictly speaking, the total number of feedback slots may be little more than that on account of overheads, and since the number of feedback bytes may not be multiples of slot lengths, and we may need ceiling operations to take this into account. However, such deviations are negligible for all practical values of $k$. 

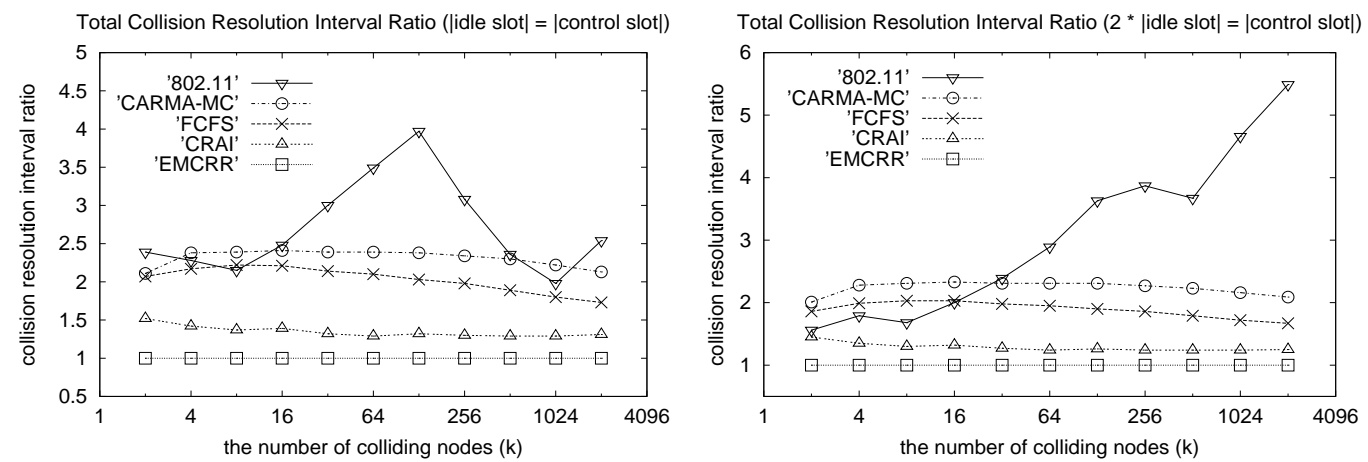

Figure 2. Average collision resolution interval length normalized that of EMCRR.
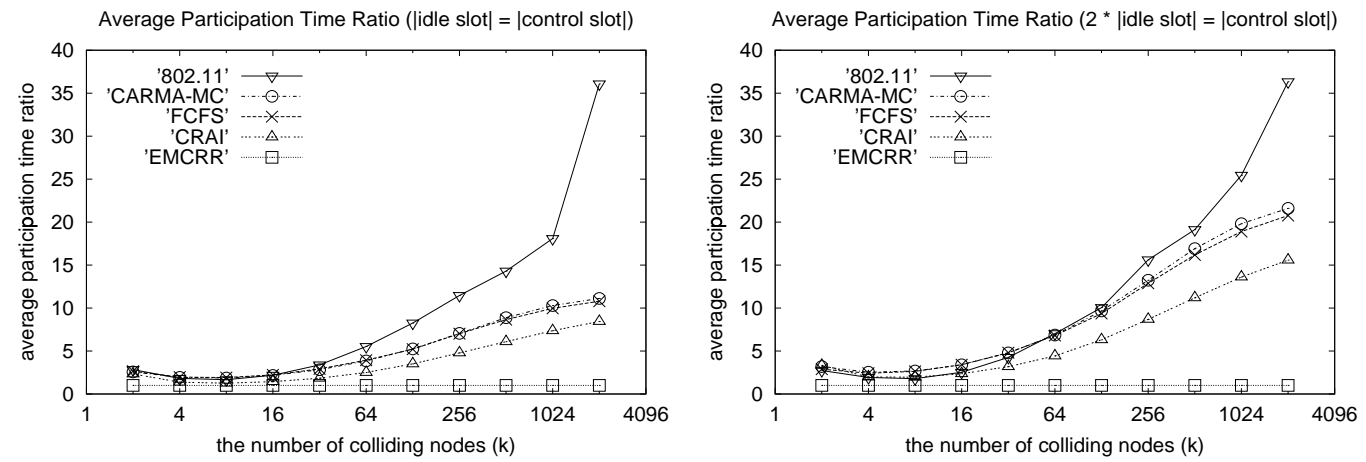

Figure 3. Average participation time normalized to that EMCRR.

collision resolution round. It follows that a transmitter needs to participate in at most $3 / 40 k+4.36 \ln k+3$ slots with a high probability. Thus the average participation time of a transmitter is upper bounded by $3 / 40 k+4.36 \ln k+3+\epsilon$ for all large $k$ and arbitrarily small $\epsilon>0$. Again, the additive constants can be ignored for large $k$.

We argued that $Z_{i}+1$ is the number of transmissions of transmitter $i$. Thus the average number of transmissions is $1+(\mathrm{E} Z / k)$. Since $\mathrm{E} Z \leq e k$, this number is upper bounded by $(e+1)$ for any $k$.

\section{Simulation Results}

We present the evaluation of our policy through simulations in this section. We evaluate our policy with respect to the total collision resolution time, the average participation time of the transmitters, the average number of transmissions and the power consumed in the collision resolution process. We study the performance for different number of contenders $(k)$. We assume that the transmitters start contending at the beginning of every reception cycle and continue till their collisions are successfully resolved (we do not allow a contender to join midway or drop out before its collision is resolved).

We have analyzed EMCRR assuming that the idle slots have the same length as the control slots. However, in many systems the receiver can detect that a slot is idle and 

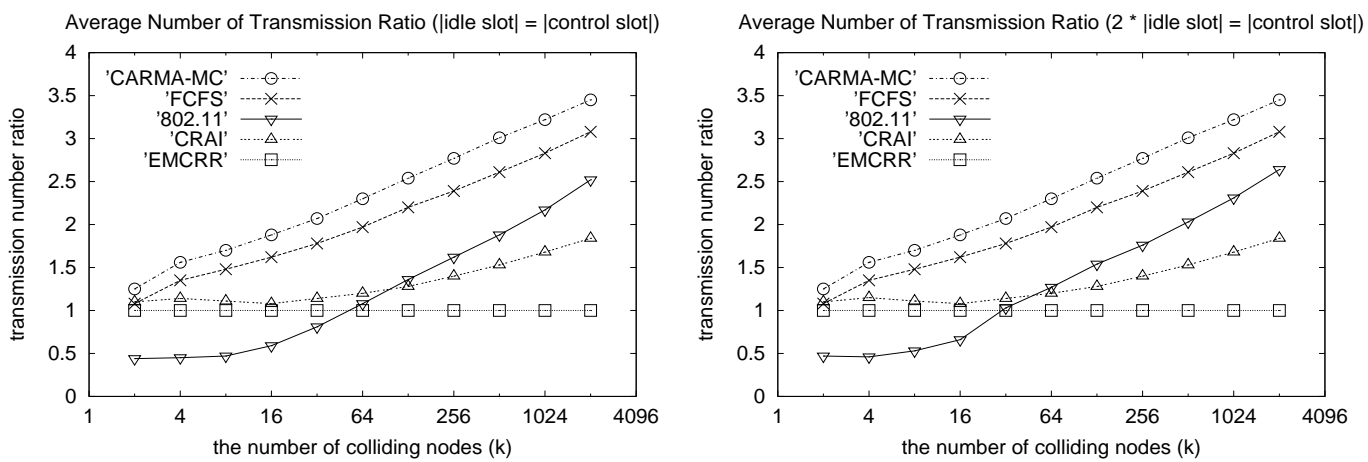

Figure 4. Average number of transmissions normalized to that of EMCRR.
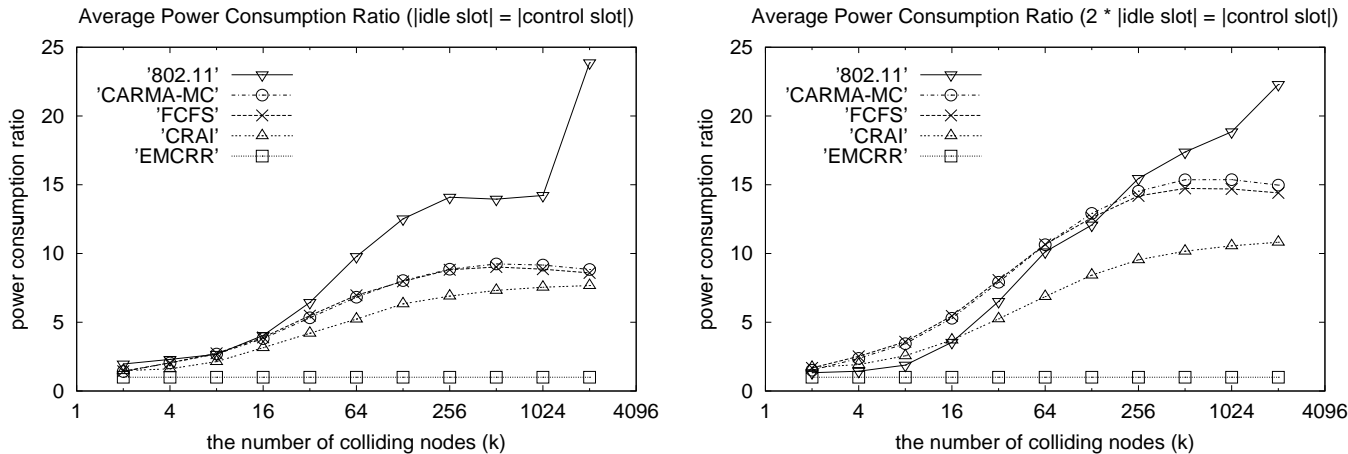

Figure 5. Average power consumption normalized to that of EMCRR.

subsequently send a feedback indicating that the slot is over. Depending on the capacity of the wireless medium, idle slots can be shorter than the control slots. In IEEE 802.11b specification that most currently available wireless interfaces follow, the idle slots can have half the length as it takes to transmit a control message (i.e., an RTS). We have experimentally studied the performance of EMCRR w.r.t. that of other approaches in this case as well. In this case, EMCRR still assumes that the idle slots have the same length as the control slots, whereas other systems terminate the idle slots faster. EMCRR is significantly better than the others even for this case. We present comparisons of EMCRR with the existing approaches, IEEE 802.11[9], FCFS[2], CRAI[5], CARMA-MC[6]. We observe that only IEEE 802.11 is sensitive to the length of the idle slots.

Figure 2 compares the average collision resolution intervals. EMCRR outperforms the other approaches due to the successful usage of the number of collisions in each slot. Figure 3 compares the average participation time of the transmitters. EMCRR also performs bettern than the other approaches. For EMCRR a transmitter knows the exact slots in which it would transmit or receive. It transmits in a randomly chosen slot every round and receives feedback at the end of the round. The length of the rounds are also known. Thus the transmitter can sleep in between. This explains the low average participation time of a transmitter in EMCRR. Figure 4 compares the average number 
of transmissions. IEEE 802.11 has fewer average transmissions than EMCRR for small values of $k(k<64)$ but this number rapidly increases with increase in the number of contenders. EMCRR has fewer average number of transmissions as compared to all the other approaches. Also, for EMCRR, this number is upper bounded by $e+1$ for all $k$, whereas the average number of transmissions increase with an increase in the number of contenders for all other approaches. Figure 5 compares the power consumption. EMCRR improves the power consumption. The significant improvement is mainly because of much smaller average participation time for EMCRR.

\section{Conclusion}

We have proposed a new energy measurement based collision resolution approach, called EMCRR. We identified some relevant performance evaluation metrics and evaluated the performance of EMCRR w.r.t. these metrics. Analytical and Experimental performance evaluation indicate that EMCRR significantly outperforms all existing approaches. In addition, EMCRR is computationally simple and does not involve sophisticated node functionality. There are several possible directions of future work. First, we would like to investigate the performance of EMCRR when nodes join and leave the collision resolution process in between. Next, we implicitly assumed that the receiver can accurately estimate the number of contending transmitters every time there is a collision. We would like to mention that similar assumptions have been made elsewhere as well[5]. In practice, the estimation is inaccurate on account of noise and imprecise power control. The study of the error resilience of EMCRR remains a topic of future research.

\section{References}

[1] B. Miller, C. Bisdikian, A. Edlund, Bluetooth Revealed: The Insider's Guide to an Open Specification for Global Wireless Communications, Prentice-Hall, 2000.

[2] D. Bertsekas and R. Gallager, Data Networks, Englewood Cliffs, NJ:Prentice-Hall, 1987.

[3] I. Capetenakis, "The Multiple Access Broadcast Channel: Protocol and Capacity Considerations", IEEE Transactions of Information Theory, pp. 505 - 515, 1979.

[4] L. Feeney, "Investigating the Energy Consumption of an IEEE 802.11 Network", SICS Technical Report SICS-T-99/11-SE, Swedish Institute of Computer Science, December, 1999.

[5] L. Georgiadis and P. Papantoni-Kazakos, "A Collision Resolution Protocol for Random Access Channels with Energy Detectors", IEEE Trans. on Communications, vol. 30, No. 11, 1982.

[6] R. Garces and J.J. Garcia-Luna-Aceves, "Collision Avoidance and Resolution Multiple Access for Multichannel Wireless Networks", Proceedings of IEEE INFOCOM 2000, Tel-Aviv, 2000.

[7] J.J. Garcia-Luna-Aceves and J. Raju, "Distributed Assignment of Codes for Multihop Packet-Radio Networks," Proceedings of IEEE MILCOM '97, Monterey, CA, November, 1997.

[8] F. Hayes, "An Adaptive Technique for Local Distribution", IEEE Transactions on Communications, Vol.26, 1978, pp $1178-1186$.

[9] IEEE, "IEEE Std 802.11b - Wireless LAN Medium Access Control(MAC) and Physical Layer(PHY) specifications: High Speed Physical Layer (PHY) in the 2.4 GHz Band", IEEE Standard 802.11, 1999.

[10] S. Khanna, S. Sarkar, and I. Shin, "An Energy Measurement Based Collision Resolution Protocol" Tech. report available at http://www.seas.upenn.edu/ swati/publication.htm

[11] J. G. Proakis, Digital Communications, McGraw-Hill, 2000.

[12] T. S. Rappaport, Wireless Communications: Principles and Practice, Prentice-Hall, 1996. 